\title{
Coaxial interleaved stimulation of the thalamus and subthalamus for treatment of Holmes tremor
}

\author{
Hiroki Toda, MD, PhD, 1,2 Namiko Nishida, MD, PhD,1 and Koichi Iwasaki, MD, PhD1 \\ ${ }^{1}$ Department of Neurosurgery, Tazuke Kofukai Medical Research Institute and Kitano Hospital, Osaka; and \\ 2Department of Neurosurgery, Fukui Red Cross Hospital, Fukui, Japan
}

Holmes tremor is often treated with multiple deep brain stimulation (DBS) electrodes. The authors describe a novel technique to suppress the tremors by effectively utilizing a single electrode.

A 16-year-old boy presented with severe right arm tremor following a midbrain injury. A DBS electrode was implanted into the ventral oralis nucleus of the thalamus (VO) and the subthalamic region. While individual stimulation of each target was ineffective, an interleaved dual stimulation of both targets has been effective for 6 years.

Coaxial interleaved stimulation of the $\mathrm{VO}$ and the subthalamic region is useful for treating Holmes tremor.

The video can be found here: https://youtu.be/tSwGh3vy68c.

KEYWORDS Holmes tremor; deep brain stimulation; thalamus; subthalamus 